\section{Deliberate self-harm and antidepressant drugs}

\author{
Investigation of a possible link
}

\author{
STUART DONOVAN, ANDREW CLAYTON, MIN BEEHARRY, SHERON JONES, \\ CHRIS KIRK, KEITH WATERS, DAVID GARDNER, JUNE FAULDING \\ and RICHARD MADELEY
}

\author{
Background It is not clear if the \\ frequency of deliberate self-harm (DSH) is \\ the same in patients taking different \\ pharmacological classes of antidepressant \\ drugs.
}

Aims To compare the frequency of DSH in patients who had been prescribed a tricyclic antidepressant (TCA) or a selective serotonin reuptake inhibitor (SSRI) prior to the DSH event.

Method This was a prospective study in 2776 consecutive DSH cases attending an accident and emergency department. The incidence of DSH inTCA-treated cases and SSRI-treated cases is expressed as number of DSH events per 10000 prescriptions of each antidepressant.

Results Significantly more DSH events occurred following the prescription of an SSRI than that of aTCA $(P<0.001)$. The occurrence of DSH was highest with fluoxetine and lowest with amitriptyline.

Conclusions Merely prescribing saferin-overdose antidepressants is unlikely to reduce the overall morbidity from DSH.

\section{Declaration of interest Support} was received from the University of Nottingham and the Southern Derbyshire Health Authority. An unconditional contribution to postage and data analysis costs was received from the pharmaceutical industry.
Tricyclic antidepressant (TCA) drugs are recognised to be inherently more toxic in overdose than the selective serotonin reuptake inhibitor (SSRI) class so that the risk of morbidity and mortality is significantly higher after TCA overdose than after SSRI overdose (Henry et al, 1995). However, this represents only part of the picture. In order to view differences between antidepressant drugs in a wider clinically relevant context, consideration needs to be given to all methods of deliberate self-harm (DSH) which occur during treatment with these drugs, not just antidepressant overdose. Several authors have addressed this question (Rouillon et al, 1989; Teicher et al, 1993; Jick et al, 1995) but with limited interpretative value. The present study aims to clarify the situation by comparing the risk of DSH by any method in a defined population of patients who had been prescribed different pharmacological classes of antidepressant drugs, particularly TCAs and SSRIs, prior to the DSH event.

\section{METHOD}

This was a prospective, observational, cross-sectional study of all consecutive cases of DSH, aged 17 years or over, who attended the accident and emergency (A\&E) department of the Derbyshire Royal Infirmary as a consequence of any act of DSH during the 2-year period between 1 January 1995 and 31 December 1996.

Data about each case were collected from two sources. Demographic and DSH event-specific data were recorded from A\&E attendance records. Data about antidepressants prescribed for depressive symptoms before the DSH event were collected from primary-care records via a postal questionnaire to the general practitioner (GP). All data were anonymised to maintain patient and GP confidentiality.

The number of prescriptions written for antidepressant drugs in the Southern
Derbyshire Health Authority region (from Prescribing Analysis and Cost (PACT) data) during the time of the study was used to calculate the incidence of DSH, expressed as number of DSH cases per 10000 prescriptions, for different antidepressant drugs. However, because not all DSH cases in this region attended the investigational site, the calculation of absolute incidences of DSH following the prescription of different antidepressants is not possible. Nevertheless, using the assumption that the available DSH case cohort is a representative sample of the whole region, the ratio of cases to prescriptions gives an incidence of DSH for different antidepressant drugs relative to one another. Incidences cited in this paper are therefore relative, not absolute.

Using the null hypothesis that there is no difference in the incidence of DSH in patients who had been prescribed different antidepressants, pairwise comparisons between the ratio of DSH events to prescriptions for each antidepressant were examined using PROC GENMOD in SAS (version 6.12 for Windows). The results are expressed as a comparison of these ratios with $95 \%$ confidence intervals and $P$-values.

\section{RESULTS}

\section{Hospital-derived data Demographics}

A total of 2776 DSH cases accrued during the 24-month case-recruitment period (mean 116 cases per month; range 98141). There were similar numbers of males $(n=1387)$ and females $(n=1389)$. The mean age of the male cases was 32.8 years (range 17-86) and that of the female cases was 33.0 years (range 17-96). For males, $27.2 \%$ fell into the age band $17-24$ years and for females the corresponding proportion was $31.0 \%$. Eight hundred and twenty-two DSH cases $(29.6 \%)$ were repeat DSH events during the time course of the study in 388 persons. Thus, during the study period, 2776 DSH events occurred in 1954 individuals. In a further 810 cases who did not repeat an episode of DSH during the study, there was a history of DSH before the start of the study. Thus, $58.8 \%$ of the total case cohort were known ever-repeaters. 


\section{Methods of DSH}

The most frequent method of DSH was overdose of a medicinal substance by any amount exceeding the maximum recommended daily dose. This occurred in $79.0 \%$ $(n=1096)$ of males and in $86.1 \%$ $(n=1196)$ of females. Other methods of DSH comprised hanging, strangulation, gassing, suffocation, drowning, inhalation, laceration, use of firearms, jumping (e.g. from high places), road traffic accident, burning, other self-violence (e.g. headbanging) and ingestion of non-medicines. Laceration was the most frequent non-overdose method of DSH in both males $(16.4 \%)$ and females $(12.6 \%)$. In a minority $(3.5 \%)$ of cases, a combination of overdose and other methods of DSH was used. Overall, there were 12 fatalities in cases who reached $A \& E$ alive (eight overdoses and four other methods). Paracetamol, either alone, or in paracetamol-containing medications or in combination with any other overdose and/or method, was the single most frequent substance taken in overdose by both males and females and comprised $39.9 \%$ of all DSH cases. Other analgesics were taken by $26.7 \%$ of cases. Antidepressant overdose, either alone or in combination with any other overdose and/ or method occurred in $16.5 \%$ of cases. Drugs of any other kind were taken in overdose by $39.9 \%$ of cases.

\section{Antidepressant overdose}

Of the $458(16.5 \%)$ cases involving antidepressant overdose, $204(44.5 \%)$ cases took one antidepressant in overdose as the sole method of DSH, 226 (49.3\%) took one antidepressant in overdose in combination with any other drug and/or method and $28(6.1 \%)$ cases took more than one antidepressant in overdose at the same time. The relative incidence of overdose with SSRIs was greater than that with TCAs (16.0 v. 11.8 cases per 10000 prescriptions respectively). This pharmacological class finding was strongly influenced by the SSRI fluoxetine, for which the relative incidence of overdose was significantly

Table I Relative risk of overdose with different antidepressants in deliberate self-harm (DSH) cases attending the accident and emergency department of Derbyshire Royal Infirmary between I January 1995 and 31 December 1996

\begin{tabular}{|c|c|c|c|c|}
\hline Antidepressant ${ }^{\prime}$ & $\begin{array}{c}\text { Overdose cases }{ }^{2} \\
\text { (n) }\end{array}$ & $\begin{array}{c}\text { Prescriptions }^{3} \\
\text { (n) }\end{array}$ & $\begin{array}{l}\text { Relative incidence (ri) } \\
\text { of overdose }{ }^{4} \text { (cases per } \\
10000 \text { prescriptions) }\end{array}$ & $\begin{array}{c}\text { Relative risk of } \\
\text { overdose }^{5}\end{array}$ \\
\hline
\end{tabular}

\section{TCAs}

\begin{tabular}{|c|c|c|c|c|}
\hline Amitriptyline & 75 & 65916 & 11.4 & 1.6 \\
\hline Clomipramine & 15 & 10911 & 13.7 & 2.0 \\
\hline Dothiepin & 51 & 51840 & 9.8 & 1.4 \\
\hline Imipramine & 12 & 17325 & 6.9 & 1.0 \\
\hline Lofepramine & 58 & 33453 & 17.3 & 2.5 \\
\hline TCAs total & 211 & 179445 & 11.8 & 1.7 \\
\hline \multicolumn{5}{|l|}{ SSRIs } \\
\hline Fluoxetine & 100 & 51538 & 19.4 & 2.8 \\
\hline Paroxetine & 40 & 30479 & 13.1 & 1.9 \\
\hline Sertraline & 13 & 13503 & 9.6 & 1.4 \\
\hline SSRIs total & 153 & 95520 & 16.0 & 2.3 \\
\hline \multicolumn{5}{|l|}{ Others } \\
\hline Flupentixol & II & 6019 & 18.3 & 2.6 \\
\hline Lithium & 13 & II 559 & 11.2 & 1.6 \\
\hline Trazodone & 9 & 7306 & 12.3 & 1.8 \\
\hline Others total & 33 & 24884 & 13.3 & 1.9 \\
\hline
\end{tabular}

Pairwise comparisons, $P<0.001$ : amitriptyline $v$. fluoxetine, ri ratio $0.59,95 \% \mathrm{Cl} 0.43-0.79$; dothiepin $v$. fluoxetine, ri ratio $0.5 \mathrm{I}, 95 \% \mathrm{Cl} 0.36-0.7 \mathrm{l}$; imipramine v. fluoxetine, ri ratio $0.36,95 \% \mathrm{Cl} 0.20-0.65$.

I. For antidepressants with at least 5000 prescriptions over the 2-year study period.

2. Antidepressant overdose either alone or in combination with any other substances and/or method.

3. Total number of prescriptions in the Southern Derbyshire region during the 2-year study period.

4. Not absolute incidence.

5. Relative to imipramine.

TCAs, tricyclic antidepressants; SSRIs, selective serotonin reuptake inhibitors. greater than that for the TCAs amitriptyline, imipramine and dothiepin (19.4 v. $11.4 v$. $9.8 v .6 .9$ cases per 10000 prescriptions respectively; $P<0.001$ ) (Table 1 ).

\section{Hospital admissions}

Out of the total cohort of 2776 cases, 1534 $(55.3 \%)$ were admitted to hospital as a direct consequence of the DSH injury. Those patients who were not admitted were either discharged from A\&E or discharged themselves against medical advice. One hundred and fifty-nine of the 204 (77.9\%) cases who took one antidepressant in overdose as the sole method of DSH were admitted to hospital. The duration of stay in hospital expressed as days per 10000 prescriptions, was greater after TCA overdose than after SSRI overdose, both for general ward admissions (7.3 v. 4.1 days per 10000 prescriptions respectively) and for intensive care ward admissions (3.6 $v$. 0.4 days per 10000 prescriptions respectively), although this difference did not reach statistical significance at these low numbers of admissions (Table 2).

\section{Primary-care-derived data}

\section{Available database}

A total of 2535 questionnaires were sent to GPs requesting information about the antidepressant prescription history of each DSH case prior to the DSH event. For $241(8.7 \%)$ cases, a questionnaire was not sent either because the identity of the GP was unknown or a repeat DSH event occurred within 1 month of a previous event. There were 1688 (66.6\%) replies, 1497 of which were evaluable for the purpose of this study. One hundred and ninety-one $(11.3 \%)$ replies were not included in the analysis either because information from the GP about that patient was not available at the time of the request or because erroneous responses on the GP questionnaire rendered the antidepressant prescription history unevaluable. Overall, evaluable data about the antidepressant prescription history prior to the DSH event were therefore available for $53.9 \%$ of the total case cohort.

\section{Antidepressant prescription history}

Five hundred and eighty-four (39.0\%) of the evaluable cases had been prescribed an antidepressant at some time during the 12 months preceding the DSH event and 186 $(31.8 \%)$ of these had overdosed on their most recently prescribed antidepressant. 
Table 2 Duration of stay in hospital following overdose with different antidepressants in deliberate self-harm (DSH) cases who attended the accident and emergency department of Derbyshire Royal Infirmary between I January 1995 and 31 December 1996

\begin{tabular}{|c|c|c|c|c|}
\hline \multirow[t]{2}{*}{ Antidepressant ${ }^{\prime}$} & \multicolumn{2}{|c|}{ General ward ${ }^{2}$} & \multicolumn{2}{|c|}{ Intensive care ward ${ }^{2}$} \\
\hline & Days (cases) & $\begin{array}{c}\text { Days per } 10000 \\
\text { prescriptions }\end{array}$ & Days (cases) & $\begin{array}{c}\text { Days per } 10000 \\
\text { prescriptions }\end{array}$ \\
\hline \multicolumn{5}{|l|}{ TCAs } \\
\hline Amitriptyline & $57(30)$ & 8.6 & $49(17)$ & 7.4 \\
\hline Clomipramine & $10(9)$ & 9.1 & $4(3)$ & 3.7 \\
\hline Dothiepin & $36(27)$ & 6.9 & $7(5)$ & 1.4 \\
\hline Imipramine & $5(4)$ & 2.9 & $4(2)$ & 2.3 \\
\hline Lofepramine & $23(20)$ & 6.9 & I (I) & 0.3 \\
\hline TCAs total (mean) & $|3|(90)$ & 7.3 & $65(28)$ & 3.6 \\
\hline \multicolumn{5}{|l|}{ SSRIs } \\
\hline Fluoxetine & $23(19)$ & 4.5 & $3(3)$ & 0.6 \\
\hline Paroxetine & $13(12)$ & 4.3 & I (I) & 0.3 \\
\hline Sertraline & $3(3)$ & 2.2 & 0 & 0 \\
\hline SSRIs total (mean) & $39(34)$ & 4.1 & $4(4)$ & 0.4 \\
\hline \multicolumn{5}{|l|}{ Others } \\
\hline Flupentixol & $5(5)$ & 8.3 & 0 & 0 \\
\hline Lithium & $5(2)$ & 4.3 & 0 & 0 \\
\hline Trazodone & $5(3)$ & 6.8 & 0 & 0 \\
\hline Others total (mean) & $15(10)$ & 6.0 & 0 & 0 \\
\hline
\end{tabular}

I. For cases where antidepressant overdose was the sole method of DSH and not confounded by a combination with other substances and/or method. Antidepressants shown are those with at least 5000 prescriptions over the 2-year period.

2. Not mutually exclusive. Some cases may spend time in both locations.

TCAs, tricyclic antidepressants; SSRIs, selective serotonin reuptake inhibitors.

The most recent prescription prior to the DSH event was for a TCA in $223(38.2 \%)$ cases, for an SSRI in 312 (53.4\%) cases and for any other antidepressant in 49 $(8.4 \%)$ cases.

However, the time interval between the most recent antidepressant prescription and the DSH event is an important consideration because the most frequent amount of antidepressant prescribed in one GP prescription is sufficient for 1 month's treatment (Donoghue \& Tylee, 1996). At prescribed doses, therefore, the patient has theoretically run out of medication after 1 month (assuming full compliance even though it is recognised that patient compliance with antidepressant medication can be poor (Maddox et al, 1994)). As a consequence, patients who commit an act of DSH more than 1 month after the most recent antidepressant prescription are arguably in a different DSH risk group (relapse or untreated risk). For this reason, only those cases whose most recent prescription for an antidepressant was 30 days or less before the DSH event (treatment risk group) are evaluated here. Three hundred and seven $(52.3 \%)$ cases met this criterion.

Pairwise comparisons of the relative incidence of DSH by any method following the prescription of named antidepressant drugs within 30 days prior the DSH event indicate that the relative incidence of any DSH event in patients who were prescribed the SSRIs fluoxetine, paroxetine and sertraline $(19.8,12.1$ and 14.8 DSH events per 10000 prescriptions respectively) was significantly higher than that in patients prescribed the TCAs amitriptyline, dothiepin and imipramine $(3.0,4.1$ and 3.5 DSH events per 10000 prescriptions respectively; $P<0.001$ ) (Table 3 ).

\section{Factors which could influence results}

There are a number of factors which may have influenced the finding from this study that the relative incidence of DSH is greater following the prescription of SSRIs than after that of TCAs.

\section{Limitations of study design}

It was assumed from the start that the case cohort in this study is representative of the whole region from which the prescription data were drawn. This assumption was not tested.

In addition, because the GP reply rate to the questionnaires was less than $100 \%$ (as described previously), there is the risk of introducing further bias due to an incomplete dataset. However, this is a factor which is not unique to this study and the evaluable reply rate in this study was in the range which might reasonably be expected for prescription event monitoring studies (Freemantle et al, 1997).

Furthermore, that this is an observational cross-sectional study rather than a randomised controlled trial leads to the question of whether patients prescribed TCAs were similar in terms of DSH risk to those prescribed SSRIs.

Each of these limitations weakens conclusions which can be drawn from this study although some of the uncontrolled DSH risk factors can be examined to estimate the likelihood of their effect on the result, as follows.

\section{Dosage of antidepressant prescribed}

Dosing below the upper daily dose recommended for use in primary care treatment (Monthly Index of Medical Specialities) was more common for TCAs $(30-75 \%$ of the upper recommended daily dose) than for SSRIs (51-125\%) particularly fluoxetine (although the tablet strength of fluoxetine makes underdosing difficult). Subtherapeutic dosing, therefore, does not appear to offer an explanation for the excess of DSH events following the prescription of the SSRIs.

\section{Duration of exposure to antidepressants}

Antidepressant drugs have a latent period before their antidepressant effect becomes maximal so that patients who have been exposed to an antidepressant for a short time may be at a different DSH risk level than those exposed for a longer time. Exposure times of the DSH cases to antidepressants prior to DSH were, however, similar for TCAs $140 \%$ of cases exposed for up to 4 weeks, $16 \%$ for $4-12$ weeks and $44 \%$ for $>12$ weeks) and SSRIs (37\%, $17 \%$ and $47 \%$, respectively). Thus, there is no evidence from this study that differences in exposure time provide an 
Table 3 Relative risk of deliberate self-harm (DSH) by any method within 4 weeks of the prior prescription of different antidepressant drugs in cases who attended the accident and emergency department of the Derbyshire Royal Infirmary between I January 1995 and 3I December 1996

\begin{tabular}{|c|c|c|c|c|}
\hline Antidepressant ${ }^{1}$ & $\begin{array}{c}\text { DSH events }{ }^{2} \\
\text { (n) }\end{array}$ & $\begin{array}{c}\text { Prescriptions }^{3} \\
\text { (n) }\end{array}$ & $\begin{array}{c}\text { Relative incidence (ri) }{ }^{4} \\
\text { of DSH (cases per } \\
10000 \text { prescriptions) }\end{array}$ & $\begin{array}{c}\text { Relative risk of } \\
\mathrm{DSH}^{5}\end{array}$ \\
\hline
\end{tabular}

TCAs

$\begin{array}{lrrrr}\text { Amitriptyline } & 20 & 65916 & 3.0 & 1.0 \\ \text { Clomipramine } & 15 & 10911 & 13.8 & 4.6 \\ \text { Dothiepin } & 21 & 51840 & 4.1 & 1.4 \\ \text { Imipramine } & 6 & 17325 & 3.5 & 1.2 \\ \text { Lofepramine } & 39 & 33453 & 11.7 & 3.9 \\ \text { TCAs total } & 101 & 179445 & 5.6 & 1.9 \\ \text { SSRIs } & & & & \\ \text { Fluoxetine } & 102 & 51538 & 19.8 & 6.6 \\ \text { Paroxetine } & 37 & 30479 & 12.1 & 4.0 \\ \text { Sertraline } & 20 & 13503 & 14.8 & 4.9 \\ \text { SSRIs total } & 159 & 95520 & 16.6 & 5.5 \\ \text { Others } & & & & 4.4 \\ \text { Flupentixol } & 8 & 6019 & 13.3 & 1.4 \\ \text { Lithium } & 5 & 11559 & 4.3 & 3.6 \\ \text { Trazodone } & 8 & 7306 & 10.9 & 2.8 \\ \text { Others total } & 21 & 24884 & 8.4 & \end{array}$

Pairwise comparisons $P<0.001$ : amitriptyline $v$. fluoxetine, ri ratio $0.15,95 \% \mathrm{Cl} 0.09-0.25$; amitriptyline $v$. paroxetine, $r$ ratio $0.25,95 \% \mathrm{Cl} 0.15-0.43$; amitriptyline v. sertraline, ri ratio $0.20,95 \% \mathrm{Cl} 0.11-0.38$; dothiepin v. fluoxetine, ri ratio $0.20,95 \% \mathrm{Cl} 0.13-0.33$; dothiepin v. paroxetine, ri ratio $0.33,95 \% \mathrm{Cl} 0.20-0.57$; dothiepin v. sertraline, ri ratio $0.27,95 \%$ $\mathrm{Cl} 0.15-0.50$; imipramine $v$. fluoxetine, ri ratio $0.17,95 \% \mathrm{Cl} 0.08-0.40$; imipramine $v$. paroxetine, ri ratio $0.29,95 \%$ $\mathrm{Cl} 0.12-0.68$; imipramine $v$. sertraline, ri ratio $0.23,95 \% \mathrm{Cl} 0.09-0.58$.

I. For antidepressants with at least 5000 prescriptions over the 2 -year study period prescribed one month or less prior to DSH.

2. DSH by any method within I month of prior antidepressant prescription.

3. Total number of prescriptions in the Southern Derbyshire region during the 2-year study period.

4. Not absolute incidence.

5. Relative to amitriptyline.

TCAs, tricyclic antidepressants; SSRIs, selective serotonin reuptake inhibitors.

explanation for the differences in the relative incidence of DSH following prescription of TCAs or SSRIs.

\section{Antidepressant prescription switches}

The prescription of different antidepressants before the most recent antidepressant is an important consideration because possible carry-over effects from the previous antidepressant cannot be ruled out, particularly when the switch was made in close time proximity before the DSH event. In those cases who had been prescribed a different antidepressant at some time before the most recent antidepressant $(n=164)$, a greater proportion of cases switched from TCAs to SSRIs (41 of 67 cases: $61 \%$ ) than from SSRIs to TCAs ( 29 of 82 cases: $35 \%$ ). This serves to suggest that SSRIs may have been more frequently prescribed when TCAs had, for whatever reason, 'failed'. Hence a greater proportion of more 'difficult to treat' patients may have been prescribed SSRIs and this may manifest as a greater risk of DSH. The prescription of some SSRIs to a higher DSH-risk group may, therefore, offer part of the explanation of the relative incidence findings of this study.

\section{Effect of co-prescribed medication}

Polypharmacy in depressed patients is not uncommon and the potential association between the co-prescription of medication other than antidepressants and the occurrence of DSH cannot be ruled out. An evaluation of the proportion of patients who were co-prescribed other psychotropic medication with antidepressants within 1 month prior to the DSH event revealed little difference between the TCAs (72 of
102 cases: $71 \%$ ) and the SSRIs (117 of 180 cases: $65 \%$ ). However, differences in the nature of the psychotropic medication co-prescribed (hypnotics, anxiolytics, antipsychotics) cannot be evaluated within the confines of this study, and therefore the potential influence of this parameter on the relative incidence findings of this study remains unclear.

\section{Influence of age}

The age of those DSH cases prescribed TCAs was higher than those prescribed SSRIs (42.3 years $v$. 33.8 years, respectively). Further exploration of this variable reveals that SSRIs were more frequently prescribed to the 17-34-years age group than TCAs (192 of 312 cases $(61.5 \%) v$. 89 of 223 cases $(40.0 \%)$, respectively). That younger age is an independent variable for the occurrence of DSH (Hawton et al, 1997) may be a contributory factor to the higher incidence of DSH seen in cases who had been prescribed SSRIs.

\section{Prior history of DSH}

An important factor in the prediction of DSH is whether it has occurred before in the same individual. In the present study, 120 of 180 cases $(67 \%)$ who had been prescribed an SSRI and 55 of 102 cases (54\%) prescribed a TCA had a known history of previous DSH at any time in the past. Thus, there is some indication from this study that SSRIs may have been preferentially prescribed to some patients who may have been at greater risk of DSH at the time of prescription by virtue of their DSH history. This suggestion makes empirical sense in that the prescription of less toxic-inoverdose SSRIs to higher DSH-risk patients would reduce complications in the event of subsequent antidepressant overdose. This factor may therefore have contributed to the finding of the greater relative incidence of DSH by any method following the prescription of SSRIs.

\section{Other potential influential factors}

There are a number of other possible factors which may have influenced the result but which are beyond the scope of exploration from the study database. These unaccountable factors include: the clinician's personal judgement of risk of DSH at the time of prescription regardless of previous DSH history; differences in the compliance of patients to taking TCAs 
or SSRIs; differences in efficacy and/or tolerability of TCAs and SSRIs in a routine primary care setting (as opposed to a clinical trial); differences in severity of depressive illness in those prescribed TCAs or SSRIs; differences in antidepressant prescription frequency for conditions other than depressive illness (e.g. chronic pain, enuresis, obsessive-compulsive disorder, weight management) which may affect the denominator (total number of prescriptions) for the relative incidence calculations to different degrees for different antidepressants; a pharmacological effect of increased suicidality in susceptible individuals.

\section{DISCUSSION}

\section{Is the present cohort representative?}

The demographic characteristics of and the DSH methods used by the present cohort are consistent with those of previously reported DSH cohorts in the UK (Smith, 1995; Bialas et al, 1996; MacNamara et al, 1996; Thomas et al, 1996; Hawton et al, 1997), indicating that the present cohort was likely to be a representative sample.

\section{Antidepressant overdose v. all methods of DSH}

It is important to appreciate the distinction between the risks associated with antidepressant overdose and the risk of any form of DSH during treatment with antidepressants at therapeutic doses. In the present study, less than one-third of DSH cases who had been prescribed an antidepressant overdosed on that antidepressant. The majority of DSH cases who had been prescribed an antidepressant harmed themselves by means other than antidepressant overdose. The finding in this study that the morbidity after TCA overdose, measured in terms of the duration of stay in hospital to effect recovery, is greater than that seen after overdose with SSRIs is not surprising, given the known overdose toxicities of these two classes of antidepressant (Henry et al, 1995). However, this study has also indicated that the risk of DSH by any method is greater in patients who had been prescribed an SSRI than in those who had been prescribed a TCA prior to the DSH event. Although not measured in this study, this gives rise to speculation that the overall morbidity may be higher after DSH in patients who had been prescribed an SSRI.

\section{CLINICAL IMPLICATIONS}

- About a third of deliberate self-harm (DSH) cases presenting to an accident and emergency department are likely to have been prescribed an antidepressant drug at some time before the DSH event and about a third of these involve antidepressant overdose as a method of DSH.

- The admission rate is higher and duration of stay in hospital is longer after tricyclic antidepressant (TCA) overdose than after selective serotonin reuptake inhibitor (SSRI) overdose, reflecting the relative higher morbidity associated with TCA overdose. However, the overall frequency of DSH of any kind is higher in patients who had been prescribed an SSRI before the DSH event than in those who had been prescribed a TCA.

- The preferential prescription of safer-in-overdose antidepressants to patients who may be at higher risk of DSH reduces the risk of morbidity following antidepressant overdose but this potential advantage is offset by the higher risk of DSH by other methods.

\section{LIMITATIONS}

- This was an observational study and so prospective matching of patients in terms of DSH risk was not feasible for cases who had been previously prescribed different classes of antidepressant drugs.

- The reply rate from primary care physicians about the antidepressant prescription history of the total DSH cohort was not $100 \%$, which limits conclusions by virtue of an incomplete dataset.

- The assumption that the antidepressant prescription patterns for the whole of the Southern Derbyshire Region is representative of the geographical area of case accrual has not been tested.

STUART DONOVAN, PhD, RICHARD MADELEY, FFPHM, School of Community Health Sciences, Division of Public Health Medicine and Epidemiology, University Hospital, Queen's Medical Centre, Nottingham; ANDREW CLAYTON, MRCPsych, MIN BEEHARRY, DipNurse, SHERON JONES, DipPsychTech, CHRIS KIRK, DPSN, KEITH WATERS, DipNurse, DAVID GARDNER, BSc, JUNE FAULDING, Deliberate Self Harm Team, The Litchurch Centre, Southern Derbyshire Mental Health Trust, Derby

Correspondence: Dr Stuart Donovan, The Croft, 44 Lower Stanton Road, Ilkeston, Derbyshire DE7 4LN, UK; fax: 0115932 1453; e-mail: mercedes@redmerc.freeserve.co.uk

(First received 4 January 2000, final revision 12 June 2000, accepted I3 June 2000)

\section{Cause and effect}

It is difficult to attribute the cause of DSH behaviour to antidepressant treatment when such behaviour can also occur spontaneously during the course of depressive illness. Establishment of cause and effect for the different apparent risks of DSH associated with different antidepressants seen in this study is therefore almost impossible.
Nevertheless, although non-fatal DSH is not a proxy for suicide because DSH is not always synonymous with failed suicide, it is a risk factor (Gunnell \& Frankel, 1994). The findings of this present study are consistent with findings of previous studies which have examined the occurrence of suicide in patients who had been prescribed different antidepressants (Isacsson et al, 1994; Jick et al, 1995; Waern et al, 1996; 
Donovan et al, 1999). Each of these previous studies indicated that, for whatever reason, the frequency of suicide, by any method, was greater in patients who had been prescribed an SSRI than that in patients who had been prescribed a TCA.

\section{Preferential prescription of safer- in-overdose antidepressants?}

Several authors have speculated on possible mechanistic factors linking suicidality and different pharmacological classes of antidepressants (Kravitz, 1990; Mann \& Kapur, 1991; Möller, 1992; Montgomery et al, 1992; Power \& Cohen, 1992; Teicher et al, 1993). Equally relevant, however, is the pragmatic consideration that prescribers are heeding advice to prescribe safer-inoverdose antidepressants to patients who are perceived to be at greater risk of DSH. This effectively 'loads the dice' against antidepressants such as the SSRIs, so that this manifests as an apparent excess of selfharm behaviour in patients who had been prescribed these antidepressants. The truth is likely to be multi-factorial and probably lies somewhere between mechanistic and pragmatic explanations.

\section{Clinical relevance of results}

Recognition of patients at risk of DSH in primary care is difficult and so the prescription of safer-in-overdose antidepressants is a laudable action to lessen complications in the event of antidepressant overdose. However, in the population described in the present study, the advantage of SSRIs over TCAs in terms of reduced overdose toxicity does not extrapolate to a reduced risk of DSH by any method. Notwithstanding the possibility that those cases who had been prescribed an SSRI in this study may be in some way at a higher risk level than those prescribed a TCA, this present snapshot of the situation does not give rise to hope that the prescription of safer-in-overdose antidepressants will lessen the overall morbidity from DSH and its burden on hospital acute services. Until the situation is further clarified by future studies, the choice of antidepressant for patients at higher risk of DSH should not be based solely on overdose toxicity. The increased risk of DSH by any method in patients prescribed SSRIs should not be underestimated.

\section{ACKNOWLEDGEMENTS}

We thank the Derbyshire Royal Infirmary Accident and Emergency Department for their permission to assimilate data from the DSH cases who attended during the case-recruitment phase of this study and all the general practitioners who gave freely of their time to complete and return the questionnaires. We also acknowledge, with grateful thanks, the Southern Derbyshire Health Authority and the Prescription Pricing Authority (copyright holder) for providing the antidepressant prescription data used in this study. S.D. is also appreciative of the unconditional contribution provided by Eli Lilly, Knoll and SmithKline Beecham Pharmaceuticals to assist with the costs of postage and data analysis associated with this study.

\section{REFERENCES}

Bialas, M., Reid, P., Beck, P., et al (1996) Changing patterns of self-poisoning in a UK health district. Quarterly Journal of Medicine, 86, 893-901.

Donoghue, J. \& Tylee, A. (1996) The treatment of depression: prescribing patterns of antidepressants in primary care in the UK. British Journal of Psychiatry, 168, 164-168.

Donovan, S., Kelleher, M., Lambourn, J., et al (1999) The occurrence of suicide following the prescription of antidepressant drugs. Archives of Suicide Research, 5, 181-192.

Freemantle, S., Pearce, G., Wilton, L., et al (1997) The incidence of the most commonly reported events with 40 newly marked drugs - a study by Prescription Event Monitoring. Pharmacoepidemiology and Drug Safety, 6, I-9.

Gunnell, D. \& Frankel, S. (1994) Prevention of suicide: aspirations and evidence. British Medical Journal, 308, 1227-1233.

Hawton, K., Fagg, J., Simkin, S., et al (1997) Trends in deliberate self-harm in Oxford, 1985-1995. Implications for clinical services and the prevention of suicide. British Journal of Psychiatry, I7I, 556-560.
Henry, J., Alexander, C. \& Sener, E. (1995) Relative mortality from overdose of antidepressants. British Medical Journal, 310, 221-224.

Isacsson, G., Holmgren, P., Wasserman, D., et al (1994) Use of antidepressants among people committing suicide in Sweden. British Medical Journal, 308, 506-509.

Jick, S., Dean, A. \& Jick, H. (1995) Antidepressants and suicide. British Medical Journal, 310, 215-218.

Kravitz, H. (1990) Fluoxetine (Prozac) and suicide: causal or casual association? Suicide Research Digest, IV, 3.

MacNamara, A., Riyat, M. \& Quinton, D. (1996) The changing profile of poisoning and its management. Journal of the Royal Society of Medicine, 89, 608-610.

Maddox, J., Levi, M. \& Thompson, C. (1994) The compliance with antidepressants in general practice. Journal of Psychopharmacology, 8, 48-53.

Mann, J. \& Kapur, S. (1991) The emergence of suicidal ideation and behaviour during antidepressant pharmacotherapy. Archives of General Psychiatry, 48, 1027-1033.

MIMS Monthly Index of Medical Specialities. London: Haymarket Publishing Services. Updated monthly.

Möller, H.-J. (1992) Antidepressants - do they decrease or increase suicidality? Pharmacopsychiatry, 25, 249-253.

Montgomery, S., Montgomery, D., Green, M., et al (1992) Pharmacotherapy in the prevention of suicidal behaviour. Journal of Clinical Psychopharmacology, 12. 27S-3IS.

Power, A. C. \& Cohen, P. J. (1992) Fluoxetine and suicidal behaviour. Some clinical and theoretical aspects of a controversy. British Journal of Psychiatry, I6I, 735-74I.

Rouillon, F., Phillips, R., Serrurier, D., et al (1989) Rechutes de depression unipolaire et efficacité de la maprotiline. L'Encéphale, XV, 527-534.

Smith, T. (1995) Differences between general practices in hospital admission rates for self-inflicted injury and self-poisoning: influence of socioeconomic factors. British Journal of General Practice, 45, 458-462.

Teicher, M., Glod, C. \& Cole, J. (1993) Antidepressant drugs and the emergence of suicidal tendencies. Drug Safety, 8, 186-212.

Thomas, S., Bevan, L., Bhattacharyya, S., et al (1996) Presentation of poisoned patients to accident and emergency departments in the North of England. Human and Experimental Toxicology, 15, 466-470.

Waern, H., Beskow, J., Runeson, B., et al (1996) High rate of antidepressant treatment in elderly people who commit suicide. British Medical Journal, 313, III8. 